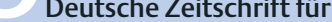 Osteopathie}

Offizielles Organ folgender Verbände

Verband der Osteopathen Deutschland e.V. (VOD)

Deutsche Akademie für Osteopathische Medizin e.V. (DAOM)

D Association Luxembourgeoise des Ostéopathes (A.L.D.O.)

ISSN 1610-5044

2003; 1 (4)

Herausgeber :

Prof. Dr. rer. nat. med. habil. Rainer Breul D.O.h.c.

Marina Fuhrmann, D.O. M.R.O.

Prof. Dr. med. habil. Karl-Ludwig Resch

Dr. med. Roger Seider, D.O.

Verlag

Hippokrates Verlag in

MVS Medizinverlage Stuttgart GmbH \& Co.KG

Oswald-Hesse-Str. 50, 70469 Stuttgart, Fax: (07 11) 8931-706 Geschäftsführung:

Dr. med. Thomas Scherb, Dipl. Kaufm. Albrecht Hauff

Programmplanung:

Marina Horbatsch

Fon/Fax: (07 11) 8931-715/-705

E-Mail: marina.horbatsch@medizinverlage.de und

redaktion.do@medizinverlage.de

Redaktion:

Christoph Newiger in team $4 \mathrm{U}$ Medienbüro

Fon/Fax: (089) 2090 01-67/-68

E-Mail: redaktion.do@medizinverlage.de

Beratung:

Dorothee Seiz

in MVS Medizinverlage Stuttgart GmbH \& Co. KC

E-Mail: redaktion.do@medizinverlage.de

Marketing:

Marion Krubasik

Fon/Fax: (07 11) 89 31-735/-706

E-Mail: marion.krubasik@medizinverlage.de

Anzeigen:

Günter Fecke, MVS Media-Service

Fon/Fax: (07 11) 8931-714/-706

E Mail: guenter.fecke@medizinverlage.de

Abonnenten-Service

Fon/Fax: (07 11) 8931-321/-422

E Mail: kundenservice@thieme.de

Produktion

Satz und Gestaltung: Fotosatz Sauter, Donzdorf

Druck: Rondo Druck, Ebersbach

Autorenhinweise

Auf Anfrage bei der Redaktion.

Urheberrecht:

Mit der Annahme eines Manuskriptes erwirbt der Verlag für die Dauer der gesetzlichen Schutzfrist ( $\$ 64$ UrhRG) die ausschließliche Befugnis zur Wahrnehmung der Verwertungsrechte im Sinne der $\S \S 15 \mathrm{ff}$. des Urheberrechtsgesetzes. Die Zeitschrift und alle in ihr enthaltenen einzelnen Beiträge und Abbildungen sind für die Dauer des Urheberrechts geschützt. Jede Verwertung ist ohne Zustimmung des Verlages außerhalb der engen Grenzen des UrhRG unzulässig und strafbar. Dies gilt insbesondere für Vervielfältigungen, Übersetzungen, Microverfilmungen und die Einspeicherung und Verarbeitung in elektronischen Systemen. Für den persönlichen Gebrauch dürfen von Beiträgen oder Teilen von diesen einzelne Kopien hergestellt werden. Die Rechte an den Abbildungen liegen

- wenn nicht anders gekennzeichnet - beim Verlag.

Erscheinungsweise:

Vierteljährlich

Bezugspreise

$\begin{array}{llll} & \begin{array}{l}\text { Abonnement- } \\ \text { preis }\end{array} & \begin{array}{l}\text { Versand- } \\ \text { kosten }\end{array} & \text { Gesamt } \\ \text { Inland } & € 64,90 & € 6,50 & € 71,40 \\ \text { Europa } & € 64,90 & € 11,20 & € 76,10 \\ \text { Restl. Welt } & € 64,90 & € 19,90 & € 84,80\end{array}$

Aus-, Fort- und Weiterbildung, Studenten und AIP:

$\begin{array}{llll}\text { Inland } & € 39,90 & € 6,50 & € 46,40 \\ \text { Europa } & € 39,90 & € 11,20 & € 51,10 \\ \text { Restl. Welt } & € 39,90 & € 19,90 & € 59,80\end{array}$

Einzelheft $€ 18,00$ zzgl. Versandkosten ab Verlagsort. Alle Preise sind unverbindlich empfohlene Preise. Alle Preise und Versandspesen enthalten $7 \%$ MwSt.

\section{Der feine Unterschied zwischen Dürfen und Dürfen}

W as wir in Deutschland dürfen, machen wir meist an unserem ursprünglich erlernten Berufstand fest. Doch die unterschiedlichen Regelungen, denen osteopathisch tätige Ärzte, Heilpraktiker und Physiotherapeuten Rechnung zu tragen haben, bilden nur die eine Seite der Medaille: Sie schreiben nur vor, was wir - unabhängig von unserem erlernten Können - rechtlich dürfen.

Gerade in einem so sensiblen Bereich, wie dem der intraanalen und intravaginalen Techniken im Rahmen der viszeralen Osteopathie gilt es, diese rechtlichen Vorgaben zu berücksichtigen. Für Physiotherapeuten ist dieser Bereich tabu.

Es gibt aber auch ein anderes „Dürfen“, das enger greift als das rechtlich Erlaubte. Es wird durch unsere Berufsethik und unseren Respekt gegenüber unseren Patienten bestimmt - gerade und vor allem bei den oben genannten Techniken. Denn wer als Patient oder Patientin einen Osteopathen aufsucht, wird normalerweise nicht erwarten, bei Verdacht auf eine entsprechende Problematik im Genital- oder Analbereich behandelt zu werden. Dabei beinhaltet schon die ganzheitliche osteopathische Untersuchung auch den Bereich des Unterleibs.

Wie also vorgehen, wenn ein internes Touché zur weiteren Untersuchung oder Behandlung notwendig erscheint?

Vier wesentliche Punkte sollten dabei zwingend berücksichtigt werden:

D Aufklärung. Führen Sie mit Ihrem Patienten vorab ein Aufklärungsgespräch. Erläutern Sie ihm, wie Sie vorzugehen beabsichtigen und warum. Ihr Patient muss wissen, was genau geschehen soll, damit er sich ohne Druck für oder gegen ein internes Touché entscheiden kann.

D Emotionale Sicherheit. Bieten Sie Ihrem Patienten die Möglichkeit, eine Person seines Vertrauens zur Behandlung mitzunehmen oder die Behandlung bei einem gleichgeschlechtlichen Therapeuten fortzusetzen. Ist eine zusätzliche Person bei der Behandlung anwesend, dient dies auch Ihrer eigenen Rechtssicherheit.

- Entscheidungsfreiheit auch während der Behandlung. Machen Sie Ihrem Patienten während des Aufklärungsgesprächs klar, dass er jederzeit die Möglichkeit hat, die Untersuchung oder Behandlung abzubrechen.

- Zeit zur Entscheidungsfindung. Geben Sie Ihrem Patienten Bedenkzeit, auf Wunsch auch bis zum nächsten Behandlungstermin hin.

Und schließlich: Bleiben Sie sachlich und üben Sie äußerste Zurückhaltung, wenn Sie mit Ihrem Patienten über intraanale oder intravaginale Techniken reden. Witze, Komplimente und dergleichen sind hier völlig fehlangebracht, auch nicht in der guten Absicht, dadurch die Atmosphäre aufzulockern.

Eigentlich sollten die oben aufgeführten Punkte selbstverständlich sein, doch zeigen staatsanwaltliche Ermittlungsverfahren wegen sexuellen Missbrauchs sowie besorgte Nachfragen von Patientinnen oder deren Partnern bei Dritten nach Untersuchungen oder Behandlungen im Intimbereich, dass oftmals zumindest recht unsensibel vorgegangen wird und sich Frauen überrumpelt fühlen.

Halten wir uns also an die oben aufgeführten Vorgaben. Zu unserer eigenen Rechtssicherheit und um die gute Arbeit, die wir täglich leisten, nicht mit Negativschlagzeilen zu diskreditieren. Das sind wir als respektvolle und verantwortungsbewusste Therapeuten uns selbst, der Osteopathie und unseren Patienten schuldig.

\section{Übrigens:}

Der VOD hat zu dieser Thematik Formblätter entwickelt, die die oben aufgeführten Vorgaben ausführlich erläutern und gemeinsam mit einem Patientenblatt bei der Geschäftsstelle oder übers Internet abgerufen werden können.

Die Herausgeber

Titelbild:

E. Blechschmidt, Die pränatalen Organsysteme des Menschen.

Hippokrates 1973; Bearbeitung: Pdesign A. Page, Stuttgart 2002 